\title{
Evaluation of abnormal biomechanics of the foot and ankle in athletes
}

\author{
V.P. A. Kannus MD, PhD \\ Tampere Research Station of Sports Medicine, The UKK-Institute, Kaupinpuistonkatu 1, SF-33500 Tampere, \\ Finland
}

\begin{abstract}
Athletes often suffer from recurrent or chronic overuse symptoms of the lower extremities. During the office visit it is essential to analyse the patient's shoes, gait cycle, lower extremities and, especially, the talocrural, subtalar and more distal joints of the ankle and foot. The basic (clinical) biomechanical analysis can be supplemented by radiographs, treadmill and video analysis and mirror table (podoscope) examinations. Ideally, successful pain relief by correction of the observed abnormality with an orthotic device completes the diagnostic procedure, especially if symptoms return soon after the removal of the device. In treatment custom-made, expensive orthotics should not be prescribed for overuse symptoms without an obvious malalignment, for asymptomatic athletes with a malalignment, or for symptoms in which the causal relationship between the biomechanical abnormality and symptoms is difficult to see. Strict indications for prescription of orthotics and close cooperation between the attending physician, physical therapist and orthotist are prerequisites for obtaining good, long-lasting results.
\end{abstract}

Keywords: Athletes, biomechanics, foot, ankle, malalignments, evaluation

With the general increase in sporting activities there has been an increase in sports injuries, both from acute and from overuse trauma ${ }^{1-3}$. Frequently athletes, both competitive and recreational, suffer from recurrent or chronic overuse symptoms at the lower extremities ${ }^{3-6}$. Main attention should be paid not to the treatment of the most painful site but to the possible cause of the symptoms, such as abnormal biomechanics of the ankle and foot.

This article describes classification and examination methods for malalignments in a competitive or recreational athlete with recurrent or chronic-type overuse injury of lower extremities.

\section{Relationship between malalignments and overuse injuries}

Causal relationship between a specific type of malalignment and a specific injury has been difficult to verify ${ }^{7}$. Associations have been reported between

Address for correspondence: Dr Pekka Kannus

*Edited version of lecture given to BASM Annual Congress, November 1991

(C) 1992 Butterworth-Heinemann Ltd 0306-3674/92/020083-07

almost all athletes' overuse injuries in lower extremities and almost all abnormal biomechanical factors, which, in turn, have been closely related to each other $^{6,8-17}$ (Table 1). On the other hand, it is the author's clinical experience that some athletes suffer from severe symptoms without any clear biomechanical abnormality in their extremities, and that some in turn may show clear malalignment but absolutely no symptoms. It seems that the human locomotor system can compensate for structural deformities and adapt to different states of stress, loading and movement ${ }^{18}$. For example, the old Chinese footbinding compressed girls' feet to small, high 'hypercavus feet' but as adults these girls seemed to suffer little from the procedure ${ }^{19}$ (Figure 1).

The literature provides no prospective, conclusive evidence that correction of malalignments with orthotics, stretching or muscle training is useful in the long term, although the results of some studies have been encouraging ${ }^{20-22}$. Therefore in clinical practice it is feasible to concentrate only on the recognition and treatment of the well-documented malalignments in the lower extremities. For an interested reader, the literature provides tens of different types of faulty postures and their combinations - only at the foot level ${ }^{12,15,16,18,23-29}$.

\section{Shoe inspection}

The clinical biomechanical examination of the athlete should be started from his or her shoes. The wear

Table 1. Characteristic biomechanical abnormalities and overuse injuries in lower extremities. Almost all of the abnormalities have been related to almost all of the injuries

Abnormality Overuse injury

Leg length inequality Femoral neck anteversion Excessive Q-angle

Genu valgum/varum

Patella alta (high-riding patella)

Squinting/frog-eye/

hypermobile patella

External tibial torsion

Foot hyper-/hypopronation

Cavus/flat foot

Forefoot valgus/varus
Low back pain

lliotibial-band friction syndrome Patellofemoral pain syndrome Patellar tendinitis

Medial/lateral/anterior compartment syndrome Stress fractures

Achilles peritendinitis Tibialis posterior tendinitis Flexor hallucis longus tendinitis Plantar fasciitis 


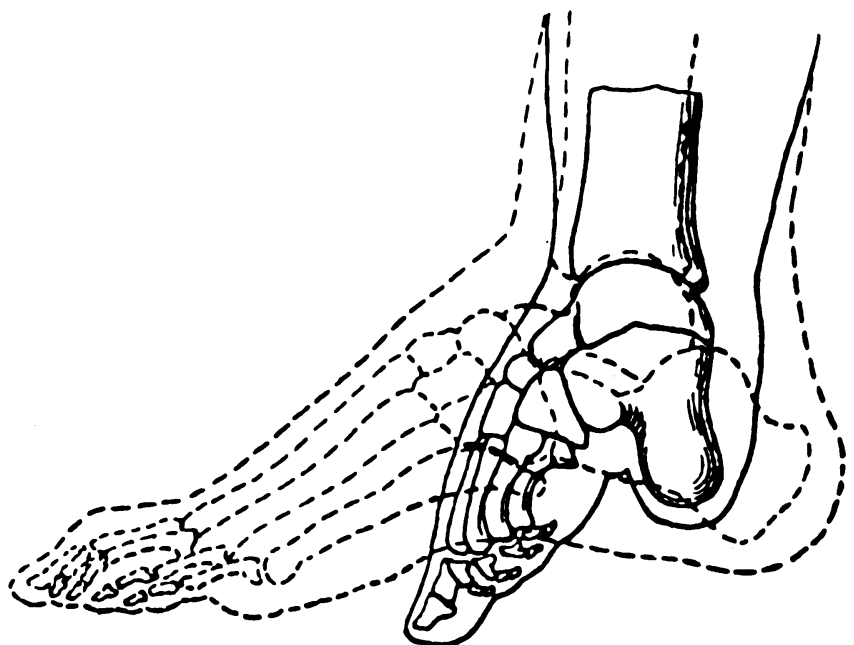

Figure 1. The old Chinese footbinding made girls' feet small and high-arched but as adults these feet were usually asymptomatic. The dotted line represents a foot of a Western woman of the same height

pattern of the athletic shoes may give a good hint of a correct biomechanical diagnosis. General shoe deformation and heel tilting to valgus is frequently found in the hyperpronation syndrome (that is, nonweightbearing forefoot varus with compensatory calcaneal valgus under weightbearing). In hypersupination (that is, non-weightbearing forefoot valgus with compensatory calcaneal varus under weightbearing) the heel tilting of the shoe is to varus.

The outersole of the shoes should be checked. Mild wearing at the lateral side of the heel and medial side of the forefoot is normal because the normal gait pressure pattern goes through these points ${ }^{29}$ (Figure 2). In hyperpronation the shoes have worn out from the medial side and in hypersupination from the lateral side, respectively.

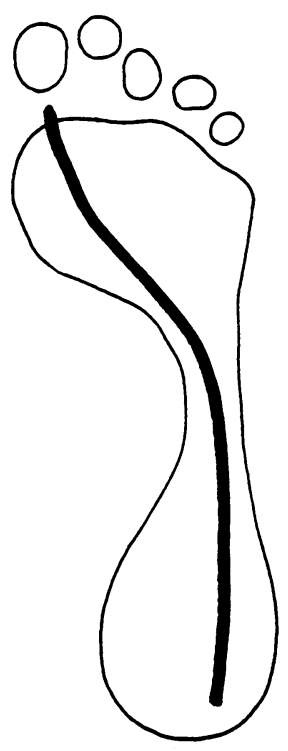

Figure 2. The normal gait pressure pattern of the foot

\section{Gait and run analysis}

The examination of the patient begins with gait analysis. Limping may be a sign of leg length inequality but often pain may be the only reason. The position of the foot in walking should be checked (abduction, adduction or straight forward). Abnormalities in foot structure and function (hyperpronation, hypersupination, flat foot, cavus foot) now become evident. Flat foot (pes planus) can be structural or functional. In the former the low arch is visible in both non-weightbearing and weightbearing conditions, in the latter only under weightbearing.

Symmetry of gait is analysed at hip, knee and ankle level in each phase - heel strike, mid-stance and push-off. A normal foot is mildly supinated at the beginning of the heel strike, pronates at the mid-stance and again supinates at the push-off phase, when it becomes a rigid lever needed for normal propulsion ${ }^{15,16,23,30}$.

In athletes, gait and running can be analysed on a treadmill using video documentation if necessary. Mild malalignments and functional variations between walking and running can be more reliably recognized from the video. A video system is also an excellent tool in revealing the efficacy of shoe orthotics in correction of the observed malalignment.

\section{General examination of the trunk and lower extremities}

General examination includes assessment of posture in all three cardinal planes ${ }^{26}$. Varus and valgus of the hip, knee and tibia; shoulder and pelvic heights; position of the patella; and foot pronation and supination are noted in the frontal plane view of the patient. The sagittal plane view allows the examiner to observe spinal curves; pelvic rotation; and hip, knee, and ankle flexion or hyperextension. Rotational components of the trunk, femur and tibia are noted in the transverse plane view of the patient. If abnormalities are found in the pelvic, hip or knee region, they should be investigated further for their possible implication in foot and ankle dysfunction.

In the examination of the lower extremities, leg length measurement is important. Traditionally, the clinical measurement of the leg length inequality (LLI) is performed by measuring from both sides the distance between the anterosuperior iliac spine and medial malleolus but its accuracy has been criticized $^{9,31,32}$.

In clinical practice, a good choice is to put lifting blocks of increasing thickness under the suspected shorter leg. Usually the patient feels no lift in the lifted shorter side until the thickness of the block exceeds the LLI. If the block is placed under the longer extremity even a 2-3-mm lift is recognized by the patient. In each phase of the procedure the examiner can compare the levels of the iliac crests by hand.

According to our experience 3-5-mm LLIs can be found by this simple clinical method. If the clinically observed LLI is over $5 \mathrm{~mm}$, we recommend to control the finding by the femoral head LLI-radiographs ${ }^{9,11,32,33}$ (Figure 3), especially if an 


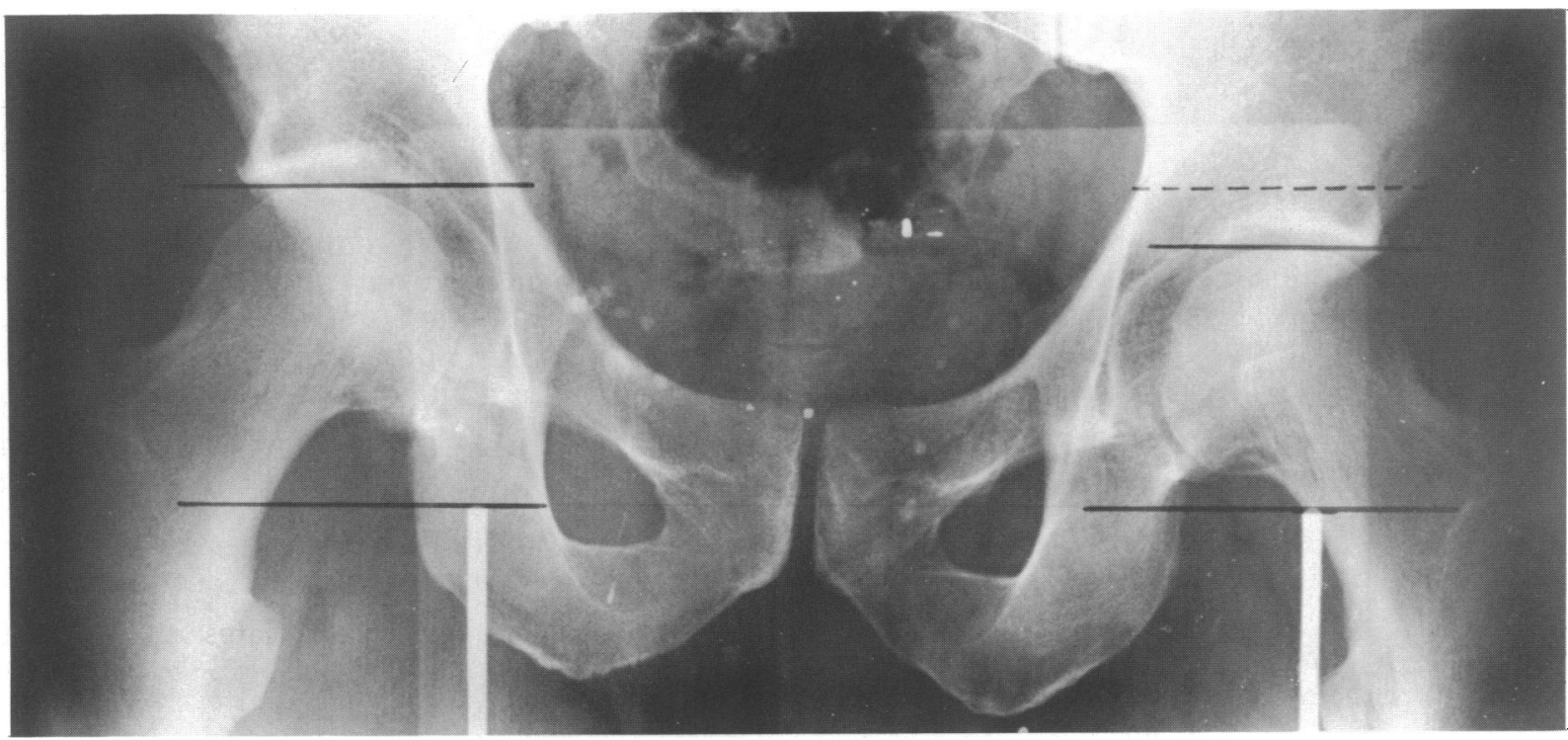

Figure 3. Leg length inequality of $12 \mathrm{~mm}$ in a 39-year-old male long-distance runner with chronic exercise-induced pain in his right knee, ankle and Achilles tendon. A 10-mm thick insert-type shoe orthotic was prescribed and the symptoms were relieved considerably within 2 weeks

orthotic shoe correction (insole or midsole) is planned. Radiographic verification of the true anatomic LLI is important since the clinically observed LLI can be functional only, due to hyperpronation and a low plantar arch of the 'shorter' leg' 15,32

General examination of the lower extremities also includes a rough evaluation of muscle strength and tightness in the major muscle groups - iliopsoas, piriformis, hamstrings, quadriceps, iliotibial band, adductors, and calf and foot extensor muscles. Running, for example, causes a relative strengthening and tightness of the gravity muscles (hamstrings and calf muscles) and weakening of the antigravity muscles (quadriceps and anterior leg muscles) which may, in turn, lead to overuse problems in lower extremities $^{15}$.

Tightness in the gastrocnemius-soleus-Achilles unit is easy to find by asking the patient to squat while keeping the back straight and feet forward. With tight calf muscles the patient cannot squat fully without lifting the heels. This test also measures ankle dorsiflexion and the tightness of the deep plantarflexors of the ankle and foot. If the calf muscles are tight, such a patient can only squat fully without lifting the heels by abducting the feet and overpronating the ankles.

\section{Examination of the ankle and foot}

The examination of the ankle and foot begins with inspection from front, sides and behind in nonweightbearing and weightbearing. If a mirror table (podoscope) is available, the footprints can be analysed. They reveal the possible flat or cavus foot, bilateral or unilateral (Figure 4). Weightbearing shows the functional deficits in the longitudinal and transversal arches. In the former the deficit is called functional flat foot. In the latter it is called weak transverse arch, which may be seen under full weightbearing as wide forefoot, often with non- specific metatarsalgia and callus formation at the ball of the foot. The toes and their malpositions (dorsi- or plantarflexed first metatarsal head, hallux valgus, hallux rigidus, and claw and hammer toes) should also be noted in this context ${ }^{30,34}$.

In athletes the main attention is paid to the supination-pronation position of the feet. By supination we mean a combination of subtalar inversion, adduction and plantarflexion; and by pronation, eversion, abduction and dorsiflexion ${ }^{16}$. In Finland approximately $40 \%$ of athletes show a static weightbearing pronation angle within normal limits $\left(4-8^{\circ}\right)$, $50 \%$ show hyperpronation $\left(>8^{\circ}\right)$ and the other $10 \%$ hypersupination ${ }^{16}$. Compared with normal feet, hyperpronated as well as hypersupinated feet do not absorb shock well ${ }^{15,30}$.

The term 'forefoot varus' means the non-weightbearing varus position of the forefoot (the great toe

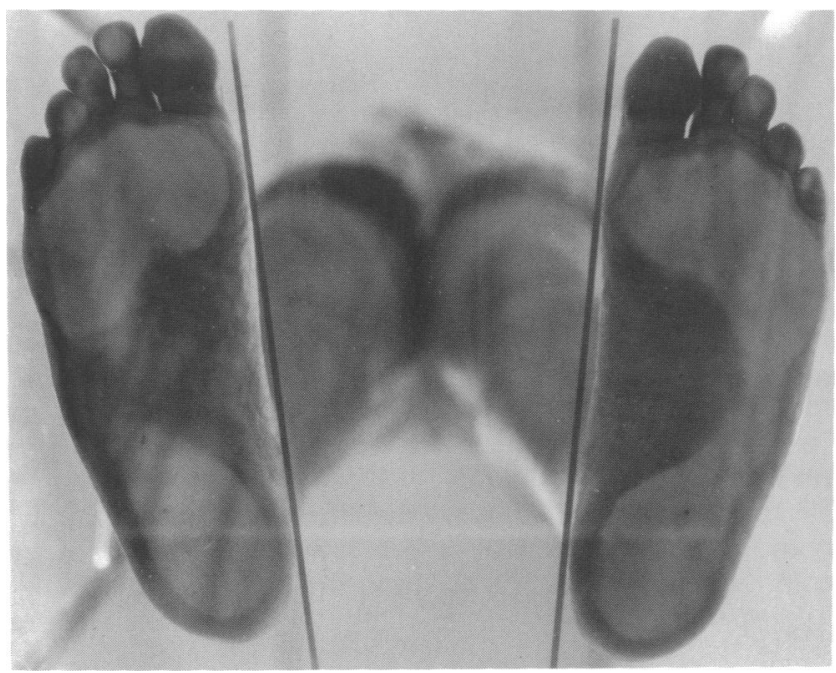

Figure 4. In a 38-year-old man unilateral (right) cavus foot abnormality becomes evident in a mirror table analysis 
and first metatarsal have risen up) while the talus and calcaneus have been placed in neutral position (that is, perpendicular to the tibia) ${ }^{16,26}$ (Figure 5a). In a normal foot, all the toes and metatarsals remain in contact with the floor. When the body weight is borne so that all toes have contact with the floor, the forefoot varus leg shows excessive (compensatory) calcaneal valgus (eversion) and the tibia rotates internally 12,16,29 (Figure 5b). In other words, the system shows ankle hyperpronation ${ }^{18}$, which can be corrected by orthotics with a medial wedge (Figures $5 c-e)$.

In the 'forefoot valgus' malposition, the little toe and fifth metatarsal bone rise up when (under non-weightbearing) the talus and calcaneus are placed in neutral position (Figure 6a). Under weight-

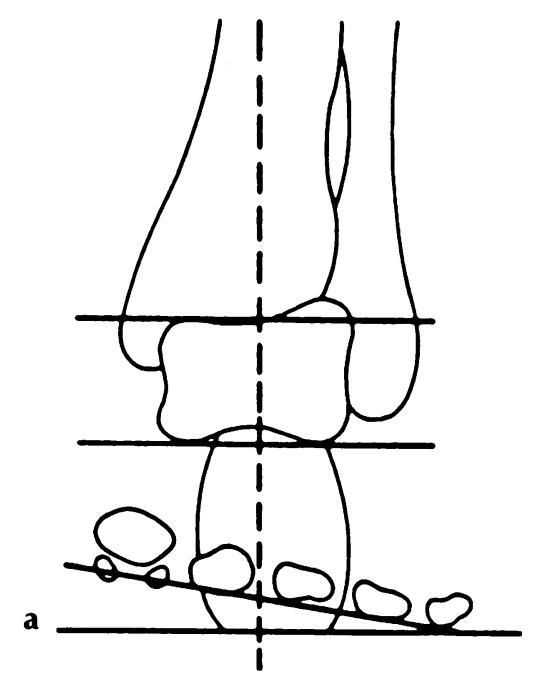

b

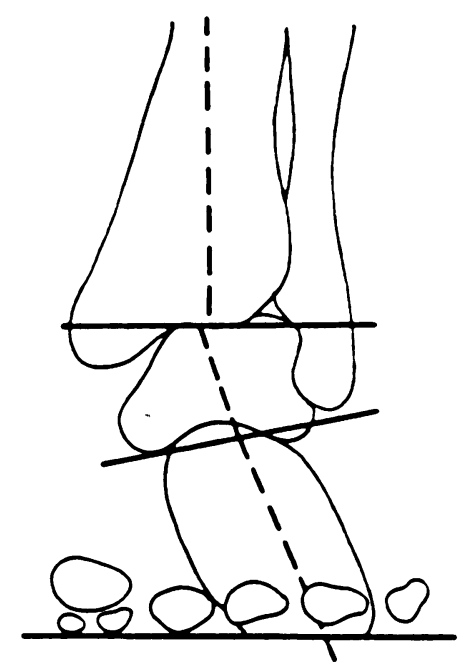

Figure 5. a In forefoot varus abnormality the great toe and first metatarsal head rise up when (without weightbearing) the talus and calcaneus are placed in a neutral position (perpendicular to the tibia). In a normal foot, all toes remain in surface contact. b Under weightbearing (when all toes have contact with the surface) there occurs a compensatory calcaneal valgus (eversion) and internal rotation of the tibia. As a whole the system shows ankle hyperpronation. c-e Under weightbearing severe bilateral ankle hyperpronation is evident in a 27 -year-old $800-\mathrm{m}$ runner. By orthotics with a medial side wedge the hyperpronation can be corrected bearing there occurs an excessive (compensatory) calcaneal varus (inversion) and the tibia rotates externally (Figure $6 b$ ). In other words, the system shows ankle hypersupination (Figure 6c).

The amount of forefoot varus (valgus) can be measured in millimetres by placing the patient prone on the examination table with the foot being examined hanging over the edge and the opposite limb flexed at the knee, and measuring the amount of the lift in the first (fifth) metatarsal head while the rearfoot is in neutral position. To get repeatable results the examiner must be well experienced. Functionally this type of static forefoot varus/valgus measurement evaluates the amount of ankle pronation-supination during loading and movement ${ }^{26}$.

In clinical practice, the pronation-supination



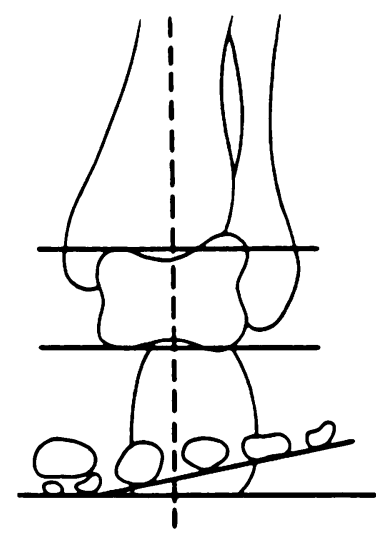

a

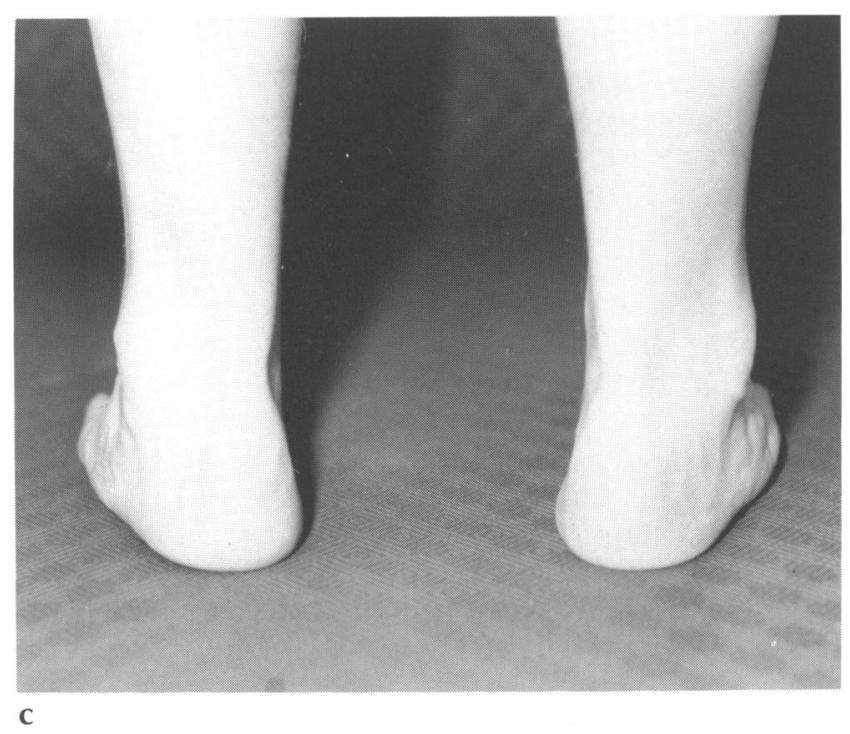

Figure 6. a In a forefoot valgus abnormality the little toe and fifth metatarsal head rise up when (without weightbearing) the talus and calcaneus are placed in a neutral position. b Under weightbearing there occurs a compensatory calcaneal varus and external rotation of the tibia. As a whole the system shows ankle hypersupination. c Ankle hypersupination syndrome in a 48-year-old recreational athlete

movement of the ankles can be more easily measured with a simple squatting test. With full weightbearing the patient squats from straight legs to $45^{\circ}$ flexion keeping the feet forward, and the examiner measures the pronation angle (degrees) with a goniometer or measures the mediolateral movement of the medial malleoli $(\mathrm{mm})$. The pronation angles achieved in this test correlate well with the dynamic videotaped treadmill recordings of ankle pronation ${ }^{10}$.

In the biomechanical analysis of the foot and ankle the clinician should always measure the range of motion of the ankle. In addition, the examination should include the talar tilt (Figure $7 a$ ) and anterior drawer (Figure $7 b$ ) tests for ankle stability since in athletes post-traumatic instability is not uncommon.

Evaluation of foot muscle strength is also part of the analysis. This screening is particularly relevant for the gastrocnemius, soleus, anterior and posterior tibialis, peroneus, and flexor and extensor hallucis longus muscles ${ }^{26}$. This includes rising up on the toes

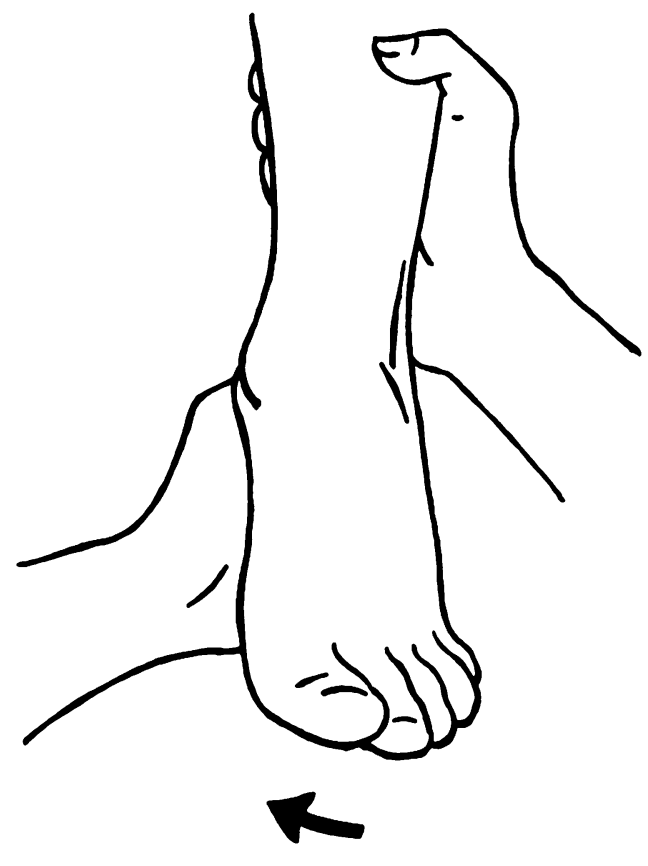

a

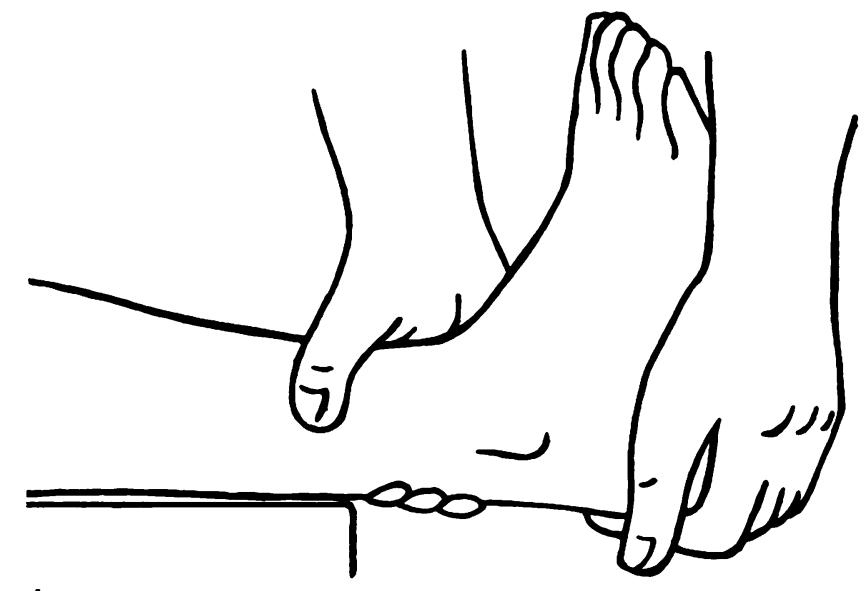

b

Figure 7. a The talar tilt test for the examination of the integrity of the calcaneofibular ligament of the ankle. b The anterior drawer test for the examination of the integrity of the anterior talofibular ligament of the ankle

rapidly in succession, walking on the heels and tiptoes, slowly lowering from a toe-rise position in succession, toe-rising slowly in an inverted and everted position, and manually resisted plantarflexion-dorsiflexion, inversion-eversion and abduction-adduction.

Balance reactions should be screened in all patients. Freeman and Wyke ${ }^{35}$ demonstrated a loss of joint mechanoreceptors with ankle injury, which may account for the patient's lack of balance. Single-limb stance with eyes shut is a much-used test for balance. The patient should be able to hold the position for at least $5 \mathrm{~s}$. A tilt board can also be used (Figure 8).

For prescription of foot orthotics, biomechanical evaluation of the foot and ankle is recommended using a mirror table. In the Tampere Research Station of Sports Medicine, Tampere, Finland the physicians send such patients to the physical therapist of the 


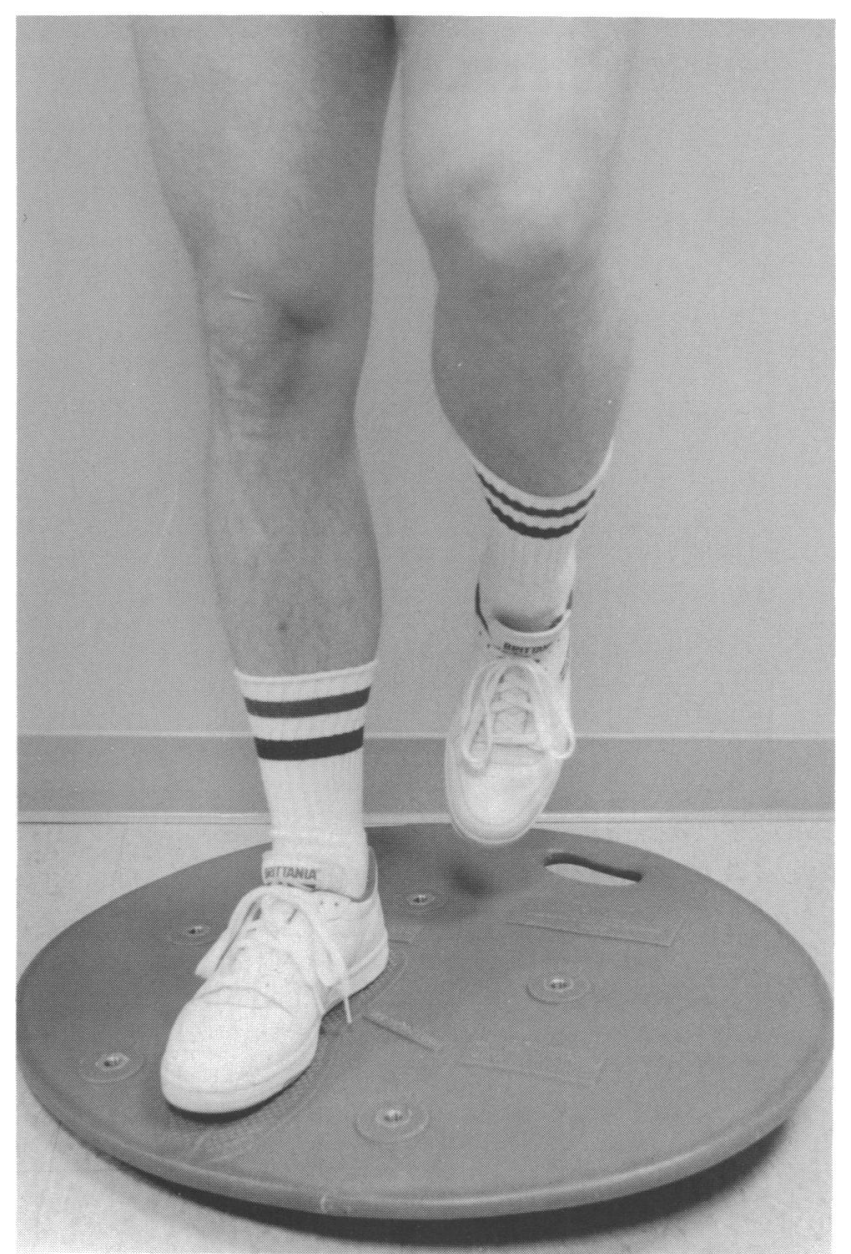

Figure 8. A tilt board is an excellent tool to test balance and ankle proprioception clinic for podoscopic foot analysis. This evaluation has six phases and the results are recorded on a standardized registration form (Figure 9). The system records the amount of forefoot varus-valgus, foot structure (flat, normal, cavus), pronation angle, mediolateral movement of the medial malleoli and the range of motion of the ankle. After this examination the therapist gives the biomechanical assessment (diagnosis) and a written recommendation for suitable orthotics or shoe correction.

\section{Correction of the malalignment as the final diagnostic tool}

Diagnosis of symptoms and observed malalignment leads to efforts to normalize foot position, i.e. to keep the subtalar joint in its neutral position under weightbearing ${ }^{12}$. These efforts consist of muscular training, stretching and foot orthotics.

Ideally, successful pain relief and resolution of other symptoms by correction of the observed abnormality with an orthotic device completes the diagnostic procedure, especially if symptoms return soon after the removal of the device. In such a case, a causal relationship between malalignment and symptoms is likely.

Causal relationships between specific malalignments and injuries have been difficult to verify. Therefore, in clinical practice it is feasible to correct the observed abnormality with orthotics only in symptomatic athletes if the causal relationship seems obvious. For example, an athlete with ankle hyperpronation and chronic tibialis posterior tendinitis may benefit from orthotics.
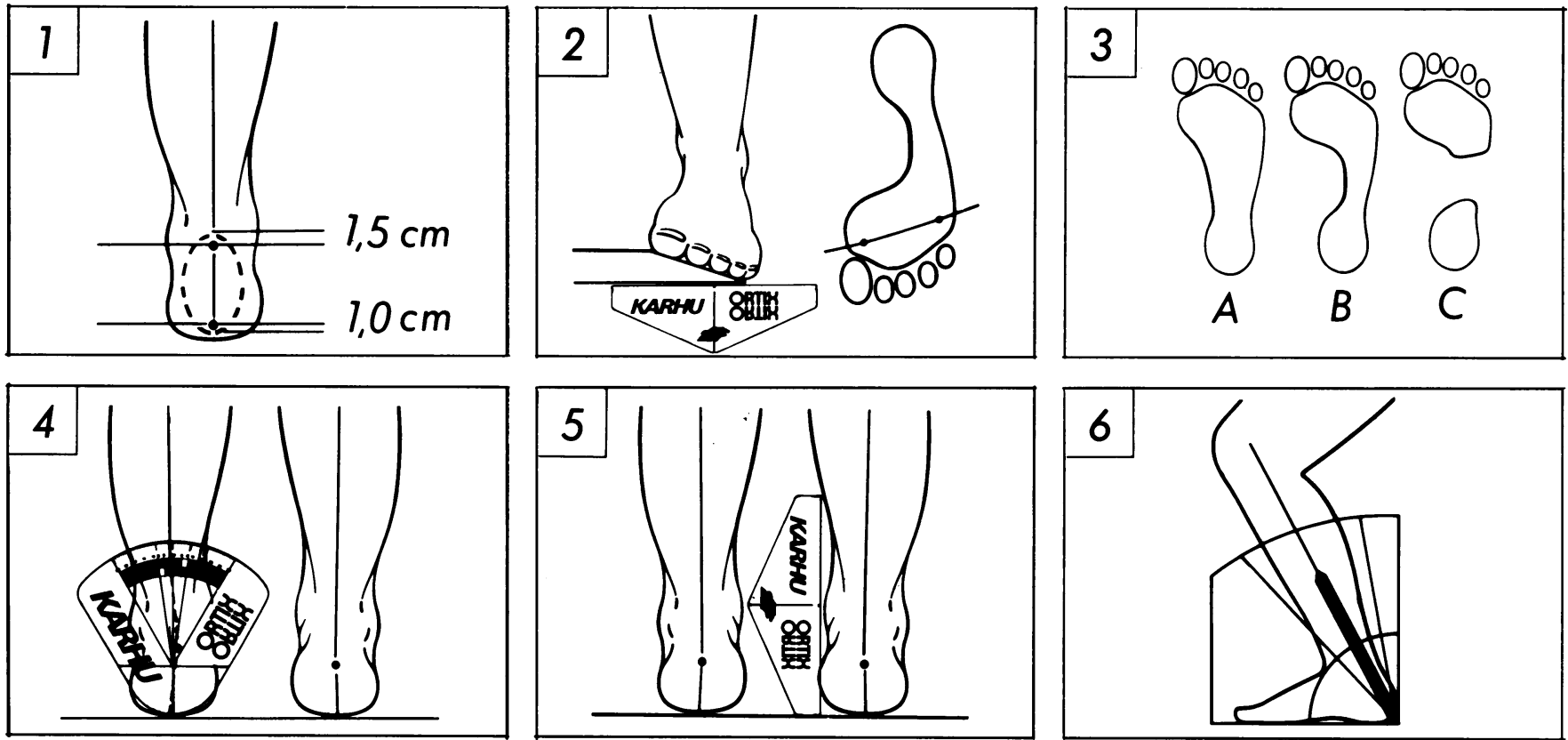

Figure 9. The six-phase podoscope examination of the ankle and foot biomechanics: (1) In a neutral position of the foot mark a straight line between the midpoints of the calf and calcaneus. (2) Measure the amount of forefoot varus/valgus in millimetres. (3) On the podoscope analyse the foot structure under weightbearing. A, flat; B, normal; C, cavus foot. (4) Under weightbearing measure the pronation angle (degrees) with a goniometer and the knees flexed $45^{\circ}$. (5) Measure the mediolateral movement of the medial malleoli $(\mathrm{mm})$ while squatting from straight legs to $45^{\circ}$ flexion. (6) Measure the range of motion of the ankle (degrees). 
On the other hand, in the treatment of athletes' overuse injuries it should be kept in mind that custom-made, expensive orthotics should not be prescribed for overuse symptoms without obvious malalignment, for asymptomatic athletes with malalignment, or for symptoms in which the causal relationship between the biomechanical abnormality and symptoms is difficult to see. Strict indications for prescription of orthotics and close cooperation between the attending physician, physical therapist and orthotist are prerequisites for obtaining good, long-lasting results.

\section{References}

1 Devereaux MD, Lachmann SM. Athletes attending a sports injury clinic - a review. Br J Sports Med 1983; 17: 137-42.

2 Walter S, Sutton J, McIntosh J, Conolly C. The aetiology of sports injuries. A review of methodologies. Sports Med 1985; 2: 47-58.

3 Kannus P, Niittymäki S, Järvinen $M$. Sports injuries in women: a one-year prospective follow-up study at an outpatient sports clinic. Br J Sports Med 1987; 21: 37-9.

4 Kvist M, Järvinen M. Typical injuries at an outpatient sports clinic. Duodecim 1978; 94: 1335-45.

5 Orava S. Exertion injuries due to sports and physical exercise. Academic Dissertation, Kokkola, Finland, 1980.

6 Torg JS, Pavlov H, Torg E. Overuse injuries in sport: the foot. Clin Sports Med 1987; 6: 291-320.

7 James SL, Bates BT, Osternig LR. Injuries to runners. Am J Sports Med 1978; 6: 40-50.

8 Taunton JE, Clement DB. Iliotibial friction band syndrome in athletes. Can J Appl Sport Sci 1981; 6: 76-80.

9 Friberg $O$. Leg length asymmetry in stress fractures. A clinical and radiological study. J Sports Med 1982; 22: 485-8.

10 Viitasalo J, Kvist M. Some biomechanical aspects of the foot and ankle in athletes with and without shin splints. Am J Sports Med 1983; 11: 125-30.

11 Friberg $\mathrm{O}$. Clinical symptoms and biomechanics of lumbar spine and hip joint in leg length inequality. Spine 1983; 8: 643-51.

12 McKenzie DC, Clement DB, Taunton JE. Running shoes, orthotics, and injuries. Sports Med 1985; 2: 334-47.

13 Renström P, Johnson RJ. Overuse injuries in sports: a review. Sports Med 1985; 2: 316-33.
14 Lindberg U. The patellofemoral pain syndrome. Linköping university medical dissertations no 227, VTT-Grafiska, Vimmerby, Sweden, 1986.

15 Subotnick SI. The biomechanics of running. Implications for the prevention of foot injuries. Sports Med 1985; 2: 144-53.

16 Santopietro FJ. Foot and foot-related injuries in the young athlete. Clin Sports Med 1988; 7: 563-89.

17 Kibler WB, Goldberg C, Chandler TJ. Functional biomechanical deficits in running athletes with plantar fasciitis. $A m \mathrm{~J}$ Sports Med 1991; 19: 66-71.

18 Tiberio D. Pathomechanics of structural foot deformities. Phys Ther 1988; 68: 1840-9.

19 Levy $\mathrm{H}$. Chinese Footbinding. The history of a curious erotic custom. London: Neville Spearman, 1966.

20 Sperryn PN, Restan L. Podiatry and sports physician - an evaluation of orthoses. Br J Sports Med 1983; 17: 129-34.

21 D'Ambrosia RD. Orthotic devices in running injuries. Clin Sports Med 1985; 4: 611-18.

22 Donatelli R, Hurlbert C, Conaway D, St Pierre R. Biomechanical foot orthotics: a retrospective study. J Orthop Sports Phys Ther 1988; 10: 205-12.

23 Brown LP, Yavorsky P. Locomotor biomechanics and pathomechanics: a review. J Orthop Sports Phys Ther 1987; 9: 3-10.

24 Donatelli R. Abnormal biomechanics of the foot and ankle. J Orthop Sports Phys Ther 1987; 9: 11-16.

25 Frey CC, Shereff MJ. Tendon injuries about the ankle in athletes. Clin Sports Med 1988; 7: 103-18.

26 Giallonardo LM. Clinical evaluation of foot and ankle dysfunction. Phys Ther 1988; 68: 1850-6.

27 Marshall P. The rehabilitation of overuse foot injuries in athletes and dancers. Clin Sports Med 1988; 7: 175-91.

28 McPoil TG, Knecht HG, Schuit D. A survey of foot types in normal females between the ages 18 and 30 years. J Orthop Sports Phys Ther 1988; 9: 406-9.

29 Nuber GW. Biomechanics of the foot and ankle during gait. Clin Sports Med 1988; 7: 1-13.

30 Bordelon RL. Practical guide to foot orthoses. J Musculoskel Med 1989; 6: 71-87.

31 Moseley CF. Leg length discrepancy. Orthop Clin North Am 1987; 18: 529-35.

32 McCaw ST, Bates BT. Biomechanical implications of mild leg length inequality. Br J Sports Med 1991; 25: 10-13.

33 Friberg O, Koivisto E, Wegelius $\mathrm{C}$. A radiographic method for measurement of leg length inequality. Diagn Imag Clin Med 1985; 54: 54-81.

34 Bordelon RL. Management of disorders of the forefoot and toenails associated with running. Clin Sports Med 1985; 4: 717-24.

35 Freeman MAR, Wyke B. Articular reflexes at the ankle joint: an electromyographic study of normal and abnormal influences of ankle joint mechanoreceptors upon reflex activity in the leg muscles. Br J Surg 1967; 54: 990-4. 